\title{
An Analysis of Health Policies Designed to Control and Prevent Diabetes in Saudi Arabia
}

\author{
Nouf Sahal Alharbi ${ }^{1,2}$, Mohammed Alotaibi ${ }^{3} \&$ Simon de Lusignan $^{1}$ \\ ${ }^{1}$ Department of Health Care Management and Policy, University of Surrey, Guildford, UK \\ ${ }^{2}$ College of Applied Studies and Community Service, King Saud University, Riyadh, Saudi Arabia \\ ${ }^{3}$ Faculty of Computers and Information Technology,University of Tabuk, Tabuk, Saudi Arabia \\ Correspondence: Simon de Lusignan, Clincal Medicine and Ageing, University of Surrey, Guildford, GU2 7HX, \\ UK. Tel: 44-1-483-368-3089. E-mail: s.lusignan@surrey.ac.uk
}

Received: January 21 Accepted: March 14, 2016 Online Published: March 31, 2016

doi:10.5539/gjhs.v8n11p233 URL: http://dx.doi.org/10.5539/gjhs.v8n11p233

\begin{abstract}
A trend analysis of the prevalence of diabetes in Saudi Arabia revealed a steep increase in diagnosis rates for the disease between the years 1989 and 2009. Between these years, the percentage of the population suffering from diabetes rose from $10.6 \%$ to $32.1 \%$ of the adult population, and the diagnosis rate is likely to increase in the future. The controlling and prevention of diabetes in the future, therefore, would potentially benefit from a scholarly review of current policies and programmes designed to contain the disease. The current study examines such policies and programmes, specifically those existing in Saudi Arabia and which are currently in operation in 2016. It employs the thematic-content-analysis technique to review key literature, and also uses Walt and Gibson's policy triangle framework to facilitate the analysis. Searches of PubMed and Medline databases were conducted to locate sources and sources were manually screened by the authors before inclusion in the study. The study concludes that prime obstacles to the successful implementation of diabetes programmes are: insufficient training of practitioners for the treatment of diabetes; lack of remuneration for the work of diabetes educators and no existing evaluation of their outputs; and a lack of training and appropriate modes of qualifying professionally for diabetes educators. The authors recommend that the Saudi government award a greater proportion of resources to programmes designed to treat diabetes sufferers, as well as educational programmes related to disease for the wider public.
\end{abstract}

Keywords: diabetes, document analysis, Saudi Arabia, policy analysis

\section{Introduction}

Diabetes is one of the most pressing health concerns facing the global population today. Both the illness itself and complications resulting from it affect millions of people living in the Gulf Cooperation Council (GCC) countries every year (Alharbi et al., 2014). It is indeed recognised by global institutions such as the World Health Organisation as the key healthcare challenge of twenty-first century. For patients and their family members, as well as for the government, the disease generates huge social and economic costs (International Diabetes Federation [IDF], 2013); see Table 1.

Table 1. Saudi Arabia at a glance (IDF Diabetes Atlas, 2013)

\begin{tabular}{lc}
\hline Adult population (aged 20-70) & $\mathbf{1 8 ~ 0 5 6 ~ 8 4 0}$ \\
Diabetes cases (aged 20-79) & $\mathbf{3 6 5 0 8 9 0}$ \\
Diabetes raw national prevalence (\%) & $\mathbf{2 0 \%}$ \\
Diabetes expenditure per diabetes patient (USD) & $\mathbf{9 4 3}$ \\
Diabetes related deaths (aged 20-79) & $\mathbf{2 2 ~ 1 1 3}$ \\
Number of people with undiagnosed diabetes (aged 20-79) & $\mathbf{1 4 8 5 9 1 0}$ \\
\hline
\end{tabular}


Understandably, then, the prevention of diabetes is fast becoming one of the key objectives of healthcare policy makers around the world (Alharbi et al., 2014). While clinical treatments of diabetes are standardised around the world, however, preventive measures need to be shaped according to localised cultures. Since societal characteristics differ widely it is thus essential to develop policies and strategies which will be effective in the context of the values and practices of the society in which they are intended to be implemented (Majeed et al., 2013).

Based on the latest Ministry of health (MOH) (2008) report, there were 1803435 registered people who have diabetes $(7.5 \%$ of the population) in Saudi Arabia. Chronic diseases, especially diabetes, pose a significant challenge to the Saudi Ministry of Health's efforts to improve health outcomes (Khoja, 2010). While the Saudi government has developed and implemented several policies designed to manage diabetes, the effectiveness of these policies has not yet been evaluated. It is therefore fitting to assess the sensitivity of these policies to the socio-cultural environment, which, as past researchers have suggested, plays a vital role in affecting the prevalence of diabetes in Saudi society. It is also important to find out if the current policies advocate the soft approach, i.e. health education and awareness, which is likely to have a more significant impact on managing diabetes than post-illness clinical care. This paper evaluates the Saudi government's policies and strategies for prevention of diabetes in Saudi society.

\section{Method}

\subsection{Study Design}

The study used Walt and Gilson's policy triangle framework, which is designed especially for analysing data produced in scientific research on health, and identifies four dimensions of understanding processes of political and cultural implementation of health policies (Figure 1) (Walt et al., 2008). The available policy papers regarding the chosen study topic were analysed using the triangle framework.

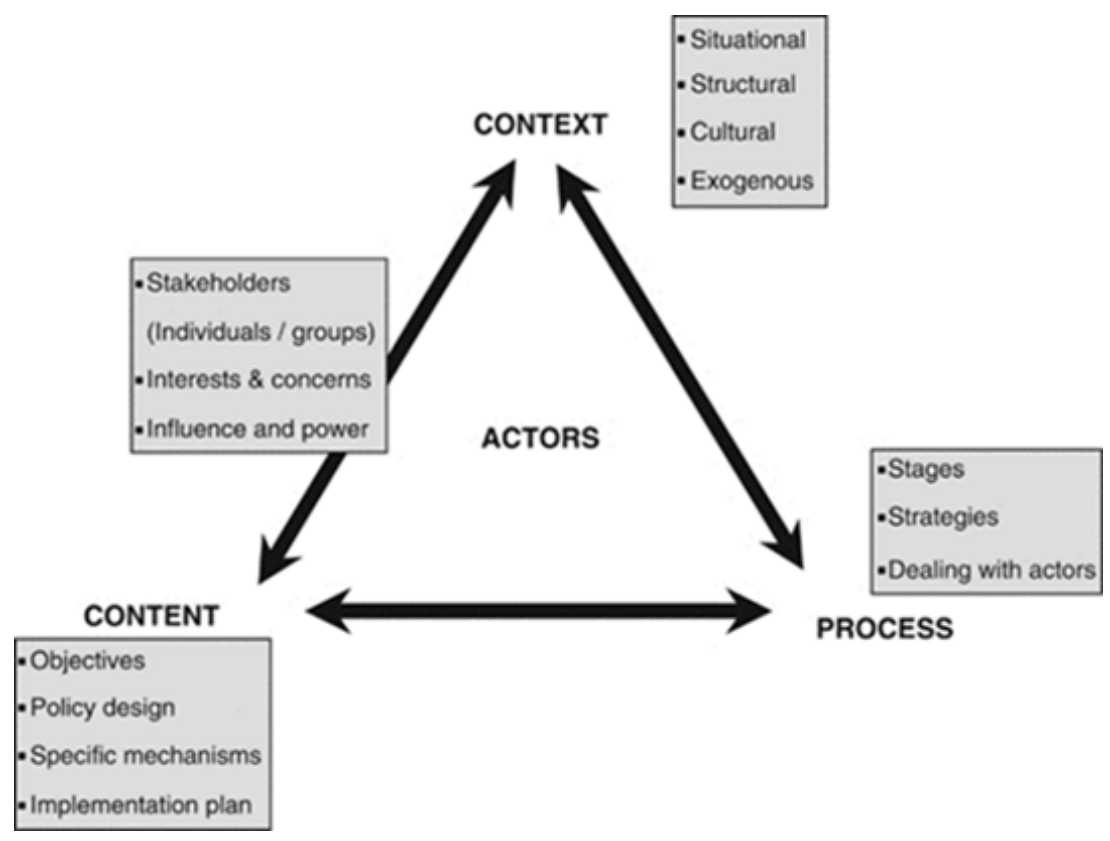

Figure 1. Model for Health Policy Analysis (Adopted from Walt and Gilson (1994))

\subsection{Search Strategy}

In conformance with qualitative methods, a combination of theory-based, convenience, and purposeful sampling strategies were adopted (Miles \& Huberman, 1994). Existing research papers on topics related to diabetes and healthcare policy were accessed. The search was limited to papers relating to GCC nations. In order to ensure reliability of secondary data, the researchers limited their scope to information provided by globally recognised institutions (such as International Monetary Fund, World Bank, United Nations Conference on Trade and Development, International Diabetes Federation, etc.) and government websites (especially GCC governments' 
websites) only. Internal websites such as the ministry of health website were also searched.

To find the required literature, several reputable online journal databases such as PubMed, Science direct, Emerald Insight, Social Science Research Network, etc. were used. In order to search for online information relevant keywords such as "healthcare policy ", "diabetes policy", "diabetes management", "diabetes control program", "diabetes control policy", "diabetes management strategy", "diabetes prevention policy", "diabetes control program", etc. were used. In order to source the most relevant information, only articles and information published in the last five years were used in this study.

The search strategy was implemented in five key stages: condensing of topic description into a single, precise sentence; highlighting keywords and phrases in the sentence; identifying synonyms and variant spelling for the keywords; linking the keywords and phrases using a Boolean search (AND/OR/NOT); and locating the information/results. This search strategy was considered appropriate for its ability to locate relevant research and allow for use of explicit methods to identify what can reliably be said on the basis of those studies. The search strategy was also seen as effective in identify, appraising, and selecting qualified research evidence, particularly in healthcare research (Bowling, 2014).

\subsection{Data Analysis}

The main aim of qualitative data analysis is to appropriately categorise it and identify dominant themes within it. In order to obtain accurate analyses, the researcher should adopt a neutral stance to the data and prevent personal views from affecting judgement. "Bracketing" is a technique which involves putting aside one's own beliefs, not making judgments about what one has observed or heard and remaining open to the data as it is revealed (Bernard and Ryan, 2010). Bracketing was used to analyse the papers.

Following the principles of thematic content analysis, the researchers classified, described and analysed relevant studies. Studies were ordered chronologically and were consulted on multiple occasions by the research team. The researchers then conducted a primary coding of all studies. The primary researcher then assigned initial codes to main themes according to each study's conceptual framework. Following this, the researchers agreed on an appropriate data analysis process after which the coding instruction was produced. This then allowed all documents to be coded and for themes to be re-classified according to the coding instruction. The codes were then reviewed again by the team and a final consensus was ascertained as to what constituted the final codes and themes.

\section{Results and Discussion}

\subsection{Content}

The Ministry of Health sought to implement objective healthcare methods in all fields of health services provision, designed to both prevent and treat patients and to rehabilitate patients. It aimed to do so by establishing a network of integrated facilities. This was with the aim of securing a decent standard of health for all. Thus, it established 20 specialized centres for treating diabetics, and the construction of eight more centres is underway across Saudi Arabia's regions and governorates. The Ministry of Health approved a ten-year national executive plan (2010-2020) for controlling diabetes and initiating future preventions (MOH, 2012).

The main, noticeable health problems in Saudi Arabia have been identified by the World Health Organisation (WHO) (2013) as an increase in non-communicable diseases (NCDs) and its modifiable behavioural risk factors. These include: tobacco use, physical inactivity and unhealthy diet. The prevalence of NCDs, diabetes, hypertension, obesity and coronary artery disease is on the rise in recent years (WHO, 2010).

The Saudi MOH and WHO both created The Cooperation Strategy for 2006-2011 (WHO, 2013) with the aim of addressing these problems. The strategy recommended that the Saudi health system should prioritize the promotion of a healthy life style, especially among young people. The strategy also suggested providing an integrated health education and research project that would be commensurate with Saudi culture. WHO (2006) identified yet another increase in diagnosis rates for chronic diseases, however, thus demonstrating that the intended aims of The Cooperation Strategy for 2006-2011 were not achieved. The same report suggested that the explanation for the failure of the initiative was a continued increase in sedentary lifestyles.

A second initiative was developed by the $\mathrm{MOH}$ focussing primarily on the promotion of a healthy lifestyle within the specific context of Saudi values and practices, thus creating a strategy that was similarly preventative in approach (WHO, 2013). It recommended an updating of all polices to ensure a more substantial implementing of health education. Several international publications (Midhet, Almohaimeed, \& Sharaf, 2010; Aldossary, Barriball, \& While, 2012; WHO, 2010; MOH, 2011) had previously argued that most major Saudi health problems are attributable to behaviour; the perceived solution, therefore, was to increase as health promotion and 
health education. It is not clear why the $\mathrm{MOH}$ created a similar strategy in light of the failure of the first initiative; it is plausible, however, that, given a high turnover of health ministers, there was little consideration of how earlier initiatives could be improved. It is certainly the case that successive ministers have simply cancelled existing initiatives

Over the last few years, the $\mathrm{MOH}$ has started several initiatives to control chronic diseases and the diabetes epidemic, including a national awareness programme for diabetes $(\mathrm{MOH}, 2013)$. These initiatives may have led to a decline in diabetes prevalence over the last eight years. Also, in September 2012, the Saudi MOH, in collaboration with the World Health Organization's Eastern Mediterranean Regional Office (EMRO), organized an international conference that aimed to address the topic of NCDs in the area (WHO Regional Office for the Eastern Mediterranean, 2012). The conference resulted in the Riyadh Declaration that included ten recommendations to combat NCDs at the regional level (WHO EMRO 2012). The MOH worked with EMRO, and the declaration was adopted by EMRO during the regional committee meeting in October 2012. This step will amount to a major impact on health in Kingdom of Saudi Arabia and the region. Indeed, the Gulf countries have similar habits and health profiles (Mokdad, Jaber, Aziz, AlBuhairan, \& AlGhaithi, 2014).

\subsection{Actors}

The Ministry of Health is the government agency responsible for healthcare policies, supervision and planning in Saudi Arabia. At the population level, the Saudi $\mathrm{MOH}$ is in charge of health promotion, early detection, and disease treatment of Saudis, and is a free healthcare system. In addition to the $\mathrm{MOH}$, there are several governmental bodies that provide health services, including the Ministry of Defence and Aviation (the second-largest health services provider), the Ministry of Interior and the National Guards. Government bodies, especially the $\mathrm{MOH}$, provide $80 \%$ of health services in the Kingdom (Alkhamis, 2012).

Since its establishment in 1951, the MOH has developed in three main stages (Regional Health Systems Observatory, 2004): during the 1970s the Ministry primarily focussed on the provision of acute care services by hospitals and tertiary health centres, and thus supported primary healthcare to a lesser extent. The second stage roughly correlates with the decade 1980-1990, which, saw WHO encourage the adoption of a 'Health for All' movement. This movement increased attention on, and recognition of, the importance of primary health care in managing health on a national basis. Therefore, early attention toward primary health services, including diabetes care, was derived from international recommendations rather than Saudi health planners' beliefs that primary care plays an essential role in improving health outcomes. In the third stage, a royal decree was issued in 2002 emphasising that health services should be provided in an equitable and affordable manner for all persons.

Actors involved with diabetes management and care in Saudi Arabia are not well placed or trained to effectively contain the disease. To best utilize its human and financial resources, Saudi $\mathrm{MOH}$ needs accurate and timely data to allocate the appropriate resources for treatment by disease or disability, but current national data on diabetes in Kingdom of Saudi Arabia are non-existent, with the most recent estimates dating from 2005 (Ministry of Health, Kingdom of Saudi Arabia, in collaboration with WHO, 2005). The data does support a number of concerns, however, one of which is a lack of human resources in the country. Saudi Arabia heavily relies on non-Arabian healthcare professionals, which make up 40 to 80 percent of the total healthcare workforce (Informa, 2012). Education on T2DM in Saudi Arabia is thus conducted by physicians alone, particularly in primary healthcare centres where there are no diabetes educators (Al-Hussein, 2008). According to a study that examined a diabetes education programme in the Asir region of Saudi Arabia, 27\% of people with diabetes received no health education and of the remaining $73 \%$ of patients who received education, only $26 \%$ of them reported receiving instructions about exercise for the managing of diabetes (Al-Khaldi \& Khan, 2000). Overall, diabetes education in Saudi Arabia does not comply with WHO's recommendations that emphasise the need for active collaboration between educators and programme recipients (Al-Khaldi \& Khan, 2000; Al-Hussein, 2008).

A study conducted in the eastern province of Saudi Arabia reported that less than half of all primary care centres attendees knew about the risk of diabetes and how to prevent it (Aljoudi \& Taha, 2009). In these centres, health education services are expected to be performed by nurses (Jradi, Zaidan, \& Al Shehri, 2012). Recent studies however show that most nurses in Saudi Arabia are expatriates from non-Arabic speaking countries and are thus unable to communicate effectively with patients (Jradi et al., 2012).

Moreover, these nurses, and even healthcare professionals speaking Arabic as their first language, were not trained in public health or health promotion (Jradi et al., 2012). In addition to the lower level of health education services, healthcare providers in Saudi Arabia do not seem to be properly advocating the measures necessary for prevention of the disease. One study was conducted to measure the effort applied by primary care physicians in Riyadh City to educate their patients in the importance of physical activity. Only $24 \%$ of physicians were 
satisfactorily engaged in such educational activity and only $45 \%$ of the physicians were properly exercising themselves. These reports together suggest a lack of a proper, public educational effort to raise awareness of diabetes (Rademeyer, 2014).

\subsection{Processes}

The Saudi public health system mainly executes diabetes healthcare through its primary healthcare service. Patients normally attend primary healthcare services before receiving referrals to specialist diabetes centres. The first stage of diabetes care is registration in a primary healthcare clinic, where patients are provided with a diabetes card. Patients are diagnosed via a medical examination and through laboratory tests on samples. Patients also receive guidance as to how to self-manage their condition, usually through use of the Diabetes Patient's Education Checklist. The goal of this first stage is to establish a diagnosis and prevent early-stage complications. The role of the diabetes centre is then to manage care and, if needed, refer patients to specialised care units, for example cardiological or surgical department. Diabetes patients are also referred by these centres for yearly physical examinations.

Treatment and control of type 2 diabetes in the Arab Gulf States was found to be suboptimal in a recent systematic review. The study based its conclusion on the three prime intermediate outcome measures (lipid control, bloody pressure and glycaemic indicators). Clinical outcome targets were met by less than $50 \%$ of sufferers (Alhyas, McKay, Balasanthiran, \& Majeed 2011). Al-Ahmadi and Roland found that primary care centres repeatedly fail to provide a sufficient standard of care, especially with regard to accurate diagnoses and referrals (Al-ahmadi, 2005). Although there is a high rate of diabetic retinopathy in Saudi Arabia, only 40-68\% of people with diabetes in the country have been referred to eye clinics. There are many reasons for this sub-optimal management of diabetes: apart from a lack of funding, primary care physicians are employed that are inadequately trained to treat diabetes; diabetes educators are rarely remunerated for their efforts or evaluated according to their professional outputs; and the same educators do not receive sufficient training and qualification. These shortcomings will be exacerbated greatly in the near future. A study by McKinsey predicted a 32\% increase in demand for diabetes treatment in GCC countries by the year 2025 (Mourshed, Hediger, \& Lambert, 2008).

There are very few papers evaluating organisational response to diabetes management and care. According to an IDF report (2014), "there is a national diabetes plan in development but no NCD plans or policies. No Ministries apart from Health are discussing the response to the diabetes challenge." Diabetes is discussed in the context of several other, categorised health issues: for example, in nutrition documents issued by Ministry of Health which emphasize the need for adequate nutrition as a means of reducing the likelihood of non-communicable diseases. Saudi Arabia has not adopted the Global Monitoring Framework for diabetes. In fact the latest IDF (2014) report suggests that the Saudi MOH does not use any framework for the monitoring and surveillance of diabetes. According to IDF report, "the health system provides services for early diagnosis and treatment, although not universally. Less than $50 \%$ of the costs are covered and no services exist for diabetes prevention. Specialised services are provided for women but availability of self-education management is limited."

\subsection{Context}

Saudi Arabia is distinct from Western societies in several ways and consequently requires policies which are in line with the local socio-cultural environment. The Kingdom of Saudi Arabia has witnessed a demographic shift over the last 20 years, accompanied by behavioural changes such as an increase in calories, fat and carbohydrate intake with a reduction in physical activity (Al-Hazzaa, Abahussain, \& Al-Sobayel, 2011; Ng, Zaghloul, \& Ali, 2011). In 2010, the country had a high proportion of years lost to disability (YLDs) caused diabetes: about $8 \%$, compared to ischemic heart disease, which possesses a YLDs percentage of $0.81 \%$. Alhyas, McKay, Balasanthiran and Majeed (2011) have characterised recent changes in Saudi culture as "rapid economic development and urbanisation; changes in lifestyle that have led to reduced levels of physical activity, increased intake of refined carbohydrates, and a rise in obesity" (Majeed et al., 2013).

Whereas WHO defines health according to personal wellbeing, sociability and productivity, Saudis tend to define it according to religious and cultural laws and traditions. In 1984, WHO defined health as "a state of complete physical, mental and social well-being and not merely the absence of disease or infirmity". Laverack (2007) states that the WHO's definition and concept of health is the most commonly used official interpretation for health promotion and health education practice. The framework considers multiple dimensions of well-being, including physical, social and mental well-being, as well as the absence of illness. Physical well-being means the healthy function of the human body, biological normality, and physical fitness and capacities to perform tasks. Social well-being incorporates a wide scope of social responsibilities and issues such as marital satisfaction, 
employability, and interpersonal relationships. Mental well-being includes self-efficacy, subjectivity well-being, social inclusion, and the abilities of individuals to adapt to their environment and their societies. Such a comprehensive definition of health would be foreign to most Saudi citizens, however, who primarily view health and wellbeing as arising from following cultural 'rules' and family traditions, and shunning technology and innovation. To be healthy is to follow the law according to Allah and illness is sent by Him; new technology has no place at present in this ancient culture. Similarly, Abdoli et al. showed that Iranian Muslims believed diabetes to be God's Will, but they also believed the body was a gift from God; therefore they had a responsibility to take care of God's gift. This study hopes to contribute to a change in beliefs using the accepted cultural norms so that the whole population of KSA can benefit from new medical knowledge and technology and thus hopefully some of the older, more dangerous practices can be eliminated.

Social and cultural practices in Saudi Arabia have a particularly direct influence in the form of traditional healing practices. Traditional medicinal practices are common in Saudi Arabia, especially among old and poorly educated Saudis in both rural and urban areas of the Kingdom (Al-Shahri, 2002). These include: herbal medicine, cauterizing, dietary treatments, chiropractic, fracture reduction, cupping, and spiritual healing (Aldosari, 2007). A study conducted by Al-Saeedi, Elzubier, Bahnassi and Al-Dawood, (2003) indicated that $15.6 \%$ of Saudis with diabetes believe that traditional herbal medicine is safe and effective for treating diabetes; $25.85 \%$ believe that traditional healers are beneficial, and $30 \%$ of patients have actually used a medicine traditionally used to treat diabetes. Al-Saeedi et al. (2003) indicate that the popularity of traditional medical practices among a large number of Saudis is very high as most of the participants have used it based on the recommendation of their friends or family members, a finding that constitutes further evidence of a widespread confidence in traditional medicine. The finding also highlights a tendency in Saudi culture for individuals to seek help from non-qualified individuals in their social circle.

A further example of confidence in traditional health practices is that Saudi girls are not allowed to play sports as it is believed that they may lose their virginity by tearing their hymens (Human Rights Watch, 2012). Even apart from this, the cultural barriers limiting even visual contact between males and females affect the ability of females in engaging in sports activities. In fact, Saudi cultural practices negatively affect women's health more generally: several studies (Al-Nozha et al., 2004; Al Quaiz \& Tayel, 2009; Sharaf, 2010) investigated Saudi women's physical activities and concluded that cultural beliefs not only create health problems arising from a lack of physical activity but also make them at risk of developing other health problems. Community support in encouraging all-round healthy lifestyles is this essential for facilitating diabetes management in Saudi Arabia.

Switching the focus to the so-called 'modern' aspects of contemporary Saudi life, there are also aspects of its current political set-up which hamper effective diabetes management. A lack of administrative stability has had a detrimental effect on the realization of government-initiated goals designed to manage diabetes. Since 2013, 6 different people have assumed the position of Minister of Health, and thus given the lack of sustained leadership from any one figure it is difficult for any one initiative to be extensively implemented.

\section{Conclusion}

In 2010, diabetes ranked fifth among leading causes of death in the Arab World, an increase from the 11th cause, as reported for 1990 (Mokdad et al., 2014). Having reviewed existing literature regarding diabetes in KSA, the study found state healthcare provisions in the country to be lacking. Primary care services are particularly underdeveloped, specifically in terms of their making accurate diagnoses and referrals. Furthermore, primary-care physicians lack adequate training regarding the disease. Diabetes educators lack specialized training and formal qualification, are rarely reimbursed for their efforts, and the results of their efforts is not monitored. The state health service is also understaffed, with a high proportion of practitioners coming from overseas. Current understanding of the disease is lacking: for example, there are no national databases focussing on diabetes. Further research on the disease is required to assist in successful containment of diabetes in the future: the Saudi $\mathrm{MOH}$ needs accurate data to allocate the appropriate resources for treatment. Bcheraoui et al. (2015) reported low utilization of free health services for preventative care, which calls for a major effort to inform the public of the value of prevention. In addition to regular physical checkups and screenings, programmes to improve diet and increase physical activity are urgently needed.

Such programmes should take into account the culture and environment in Saudi Arabia. Implementing such measures is particularly difficult in Saudi Arabia, however, where a persistent trust in cultural and religious values and practices tends to override an embracing of progressive reforms advocated by global health organisations, and where a lack of indoor spaces or amenities provided in a hot climate deters people from engaging in physical activity. Creative methods will need to be adopted to increase physical activity in a very hot 
environment. In many places in the world, outdoor activities are encouraged, but in Saudi Arabia indoor activities have to be established and made available to the public and communities, perhaps using Saudi Arabia's large indoor infrastructure, which includes malls and public spaces.

There are governmental mechanisms in place in Saudi Arabia which are currently addressing the problem of ineffective policy implementation caused by administrative instability (as previously noted, the Minister for Health has been replaced six times in the past two years). The National Authority for Combating Corruption investigates the failure of successive ministers to responsibly manage policies established by their predecessors. It aims to identify those responsible for creating delays in implementing projects and attempts to issue sanctions, with the ultimate aim of enabling citizens across Saudi Arabia to directly benefit from initiatives at the pragmatic health level.

\section{Limitations of the Study}

A lack of available data made it difficult to evaluate the extent to which interventions in diabetes cases in GCC countries are effective. Additionally, the data and findings covered by the study are not extensive or exhaustive owing to its primary focus on qualitative as opposed to quantitative studies.

\section{Ethical Considerations}

The authors have fully taken into account relevant ethical issues, including plagiarism and appropriate response to instances of data duplication or double submission of articles.

\section{Acknowledgments}

This work was part of a $\mathrm{PhD}$ dissertation, funded by King Saud University

\section{Conflict of Interest Statement}

The authors declare that there is no conflict of interest

\section{References}

Abdoli, S., Ashktorab, T., Ahmadi, F., Parvizy, S., \& Dunning, T. (2011). Religion, faith and the empowerment process: Stories of Iranian people with diabetes. International journal of nursing practice, 17(3), 289-298. http://dx.doi.org/10.1111/j.1440-172X.2011.01937.x

Al-Ahmadi, H., \& Roland, M. (2005). Quality of primary healthcare in Saudi Arabia: A comprehensive review. International Journal for Quality in Healthcare, 17(4), 331-346. http://dx.doi.org/10.1093/intqhc/mzi046

Aldosari, A. (2007). Middle East, Western Asia, and Northern Africa. New York: Marshall Cavendish.

Aldossary, A., Barriball, L., \& While, A. (2012). The perceived health promotion practice of nurses in Saudi Arabia. Health Promotion International, 28(3), 431-441. http://dx.doi.org/10.1093/heapro/das027.

Alharbi, N. S., Almutari, R., Joes, S., Al-Daghri, N., Khunti, K., \& de Lusignan, S. (2014). Trends in the prevalence of type 2 diabetes mellitus and obesity in the Arabian Gulf States: Systematic review and meta-analysis. Diabetes Research and Clinical Practice, 106(2), 30-3. http://dx.doi.org/10.1016/j.diabres.2014.08.019

Al-Hazzaa, H. M., Abahussain, N. A., \& Al-Sobayel, H. I. (2011). Physical activity, sedentary behaviors and dietary habits among Saudi adolescents relative to age, gender and region. Int J Behav Nutr Phys Act, 8, 140. http://dx.doi.org/10.1186/1479-5868-8-140

Alhyas, L., McKay, A., Balasanthiran, A., \& Majeed, A. (2011). Prevalences of overweight, obesity, hyperglycaemia, hypertension and dyslipidaemia in the Gulf: Systematic review. JRSM Short Rep, 2(7), 55. http://dx.doi.org/10.1258/shorts.2011.011019

Al-Hussein, F. A. (2008), Prescription non-conformities in primary care settings: How useful are guidelines. $J$ Family Community Med, 15(2), 51-56.

Aljoudi, A. S., \& Taha, A. Z. (2009). Knowledge of diabetes risk factors and preventive measures among attendees of a primary care center in eastern Saudi Arabia, Ann Saudi Med, 29(1), 15-9. http://dx.doi.org/10.4103/0256-4947.51813

Alkhamis, A. (2012). Health care system in Saudi Arabia: An overview. Eastern Mediterranean Health Journal, 18(10), 1078-1080.

Al-Khaldi, Y. M., \& Khan M. Y. (2000). Audit of a diabetic health education program at a large primary healthcare center in Asir region. Saudi Med J, 21(9), 838-42. 
Al-Nozha, M., Al-Maatouq, M., Al-Mazrou, Y., Al-Harthi, S., Arafah, M., Khalil, M., ... Al-Mobeireek, A. (2004). Diabetes mellitus in Saudi Arabia. Saudi Med J., 25(11), 1603-1610.

Al Quaiz, A., \& Tayel, S. (2009). Barriers to a healthy lifestyle among patients attending primary care clinics at a university hospital in Riyadh. Ann Saudi Med., 29(1), 30-35. http://dx.doi.org/10.4103/0256-4947.51818

Al-Saeedi, M., Elzubier, A. G., Bahnassi, A. A., \& Al-Dawood, K. M. (2003). Patterns of elief and use of traditional remedies by diabetic patients in Mecca, Saudi Arabia. East Mediterr Health J, 9(1-2), 99-107.

Al-Shahri, M. (2002). Culturally sensitive caring for Saudi patients. Journal of Transcultural Nursing, 13, 133-138. http://dx.doi.org/10.1177/104365960201300206

Bernard, H. R., \& Ryan. G. W. (2010). Analyzing qualitative data. Thousand Oaks, CA: Sage Publications.

Bowling, A. (2014). Research ethods in health, investigating health and health services (4th ed.). Berkshire, England: Open University Press.

El Bcheraoui, C., Tuffaha, M., Daoud, F., Kravitz, H., AlMazroa, M. A., Al Saeedi, M., ... Mokdad, A. H. (2015). Access and barriers to healthcare in the Kingdom of Saudi Arabia, 2013: Findings from a national multistage survey. BMJ Open, 12, 5(6), e007801. http://dx.doi.org/10.1136/bmjopen-2015-007801

Health system profile. Saudi Arabia. World Health Organization Eastern Mediterranean Regional Health System Observatory [online database]. Retrieved from http://gis.emro.who.int/HealthSystemObservatory/Profile/ Forms/frmProfileSelectionByCountry.aspx?CountryID=SAP000000000000000000\&CountryName=Saudia $\% 20$ Arabia

Human Rights Watch. (2012). Saudi Arabia: An Olympic Advance for Women. The International Equestrian Federation. Retrieved from http://www.hrw.org/news/2012/06/25/saudi-arabia-olympic-advance-women

Informa. (2012). Healthcare in the GCC: A Snapshot. Hospital Build \& Infrastructure Magazine. Retrieved from http://inc.iirme.com/Sites/HospitalBuild/v1/Downloads/resources/articles/Regional\%20healthcare/regional_ healthcare.pdf

International Diabetes Federation. (2013). IDF Diabetes Atlas. $(6$ th $\quad$ ed.). Retrieved from http://www.idf.org/sites/default/files/EN_6E_Atlas_Full_0.pdf.

International Diabetes Federation (2014). Diabetes: facts and figures. Retrieved from http://www.idf.org/worlddiabetesday/toolkit/gp/facts-figures.

Jradi, H., Zaidan, A., \& Al Shehri, A. M. (2013). Public health nursing education in Saudi Arabia. Journal of Infection and Public Health, 6, 63-68. http://dx.doi.org/10.1016/j.jiph.2012.11.002

Khoja, T. (2010). Strategic approaches in combating diabetes mellitus among GCC countries. Paper presented at the International Gulf Group Study for Diabetes Conference. Jeddah, Saudi Arabia.

Laverack, G. (2007). Health promotion practice: Building empowered communities. Maidenhead: Open University Press.

Mourshed, M., Hediger, V., \& Lambert, T. (2008). Gulf cooperation council health care: challenges and opportunities. Retieved from http://www.weforum.org/pdf/Global_Competitiveness_Reports/ Reports/chapters/2_1.pdf

Majeed A., El-Sayed, A. A., Khoja, T., Alshamsan, R., Millett, C., \& Rawaf, S. (2013). Diabetes in the Middle-East and North Africa: An update. Diabetes Research and Clinical Practice, 103(2), 218-22. http://dx.doi.org/10.1016/j.diabres.2013.11.008

Midhet, F., Al Mohaimeed, A. R., \& Sharaf, F. (2010). Dietary practices, physical activity and health education in qassim region of Saudi Arabia. International journal of health sciences, 4(1), 3-10.

Miles, M. B., \& Huberman, A. (1994). Qualitative data analysis: An expanded sourcebook. Thousand Oaks: Sage Publications.

Mokdad, A. H., Jaber, S., Aziz, M. I., AlBuhairan, F., \& AlGhaithi, A. (2014). The state of health in the Arab world, 1990-2010: An analysis of the burden of diseases, injuries, and risk factors. Lancet, 383, 309-320. http://dx.doi.org/10.1016/S0140-6736(13)62189-3

Ng, S. W., Zaghloul, S., \& Ali, H. I. (2011). The prevalence and trends of overweight, obesity and nutrition-related non-communicable diseases in the Arabian Gulf States. Obes Rev Off J Int Assoc Study Obes, 12, 1-13. http://dx.doi.org/10.1111/j.1467-789X.2010.00750.x 
Saudi Ministry of Health. (2011). Health statistical book. Retrieved from http://www.moh.gov.sa/Ministry/Statistics/book/Pages/default.aspx

Saudi Ministry of Health. (2011b). Nursing policy and procedural manual (2011). Retrieved from http://forum.hail-nurses.com/showthread.php?t=3168

Saudi Ministry of Health. (2012). Saudi ministry of health strategical plan. Retrieved from http://www.moh.gov.sa/Portal/WhatsNew/Documents/OKIstragi260p.pdf

Saudi Ministry of Health. (2013). Press report on the MOH's efforts regarding raising people's awareness of diabetes. Retrieved from http://www.moh.gov.sa/en/Ministry/MediaCenter/Publications/ Pages/Publications-2013-06-09-004.aspx

Sharaf, F. (2010). Impact of health education on compliance among patients of chronic diseases in Al Qassim, Saudi Arabia. Int J Health Sci, 4(2), 139-48.

Walt, G., Shiffman, J., Schneider, H., Murray, S. F., Brugha, R., \& Gilson, L. (2008). 'Doing' health policy analysis: Methodological and conceptual reflections and challenges. Health Policy Plan, 23, 308-17. http://dx.doi.org/10.1093/heapol/czn024

World Health Organization. (1984). Glossary of terms used in 'Health for All' series.

World Health Organization's. (2001). Skills for health. The Pan American Health Organization. Retrieved from http://www.who.int/school_youth_health/media/en/sch_skills4health_03.pdf

World Health Organization. (2006). Country cooperation strategy for WHO and Saudi Arabia 2006-2011. Retrieved from http://www.who.int/countryfocus/cooperation_strategy/ccs_sau_en.pdf

World Health Organization. (2010). Countryspecific standard report: Saudi Arabia. Retrieved from http://www.who.int/nmh/countries/sau_ar.pdf

World Health Organization. (2012). Health education. World Health Organization. Retrieved from http://www.who.int/topics/health_education/en/

World Health Organization. (2012). Hennessy-Hicks training needs analysis questionnaire And manual. Retrieved from http://www.who.int/workforcealliance/knowledge/resources/hennessyhicks trainingneedsquest/en/index.html

World Health Organization (2013). Country cooperation strategy for WHO and Saudi Arabia 2012-2016. Retrieved from http://www.who.int/countryfocus/cooperation_strategy/ccs_sau_en.pdf

\section{Copyrights}

Copyright for this article is retained by the author(s), with first publication rights granted to the journal.

This is an open-access article distributed under the terms and conditions of the Creative Commons Attribution license (http://creativecommons.org/licenses/by/3.0/). 